University of St. Thomas, Minnesota

UST Research Online

Marketing Faculty Publications

Marketing

2018

\title{
Lean Six Sigma and Assurance of Learning: Challenges and Opportunities
}

\author{
Richard J. Rexisen \\ University of St. Thomas, Minnesota, rjrexeisen@stthomas.edu \\ Ernest L. Owens \\ University of St. Thomas, Minnesota, elowens@stthomas.edu \\ michael j. garrison \\ University of St Thomas, mjgarrison@stthomas.edu
}

Follow this and additional works at: https://ir.stthomas.edu/ocbmktgpub

Part of the Business Administration, Management, and Operations Commons, Business Law, Public Responsibility, and Ethics Commons, and the Marketing Commons

This Article is brought to you for free and open access by the Marketing at UST Research Online. It has been accepted for inclusion in Marketing Faculty Publications by an authorized administrator of UST Research Online. For more information, please contact asle4660@stthomas.edu. 


\title{
Lean Six-Sigma \& Assurance of Learning: Challenges and Opportunities
}

https://doi.org/10.1080/08832323.2018.1457619

\author{
Richard J Rexeisen \\ University of St. Thomas \\ Mail \# MCH 316 \\ 2115 Summit Ave \\ St. Paul, MN 55105 \\ rjrexeisen@stthomas.edu \\ 651-962-5079 \\ Ernest L Owens Jr \\ University St Thomas \\ Mail \# MCH 316 \\ 2115 Summit Ave \\ St. Paul, MN 55105 \\ elowens@sthomas.edu \\ 651-962-5141 \\ Michael J Garrison \\ University of St. Thomas \\ Mail \# MCH 128 \\ 2115 Summit Ave \\ St. Paul, MN 55105 \\ mjgarrison@stthomas.edu \\ 651-962-5187
}

Correspondence should be directed to Dr. Richard Rexeisen, rjrexeisen@stthomas.edu, 651-962-5079 


\title{
Lean Six-Sigma \& Assurance of Learning: \\ Challenges and Opportunities
}

\begin{abstract}
The importance, and associated challenges, of faculty ownership and engagement in the Assurance of Learning (AoL) process are well documented in the literature. This paper demonstrates how schools can further their AoL objectives by encouraging and subsequently supporting organic, faculty led initiatives. Specifically, we present a case study of a faculty led initiative to explore and demonstrate, as a proof of concept, how Lean Six-Sigma (LSS) can be used to improve AoL practices.
\end{abstract}

Key Words: Assurance of Learning, Faculty Engagement, Lean Six-Sigma, AACSB 


\section{Introduction}

The call for greater accountability in higher education continues to grow with increasing intensity (Report to the Secretary of Education, 2012; Eaton, 2010; Millet et al, 2008) including more recent attempts to link student outcomes assessment with the evaluation of operations, planning and budgeting (Higher Learning Commission Standards, 2017, Criterion 5.C.2). While many factors contribute to the challenges of developing and implementing a cost-effective Assurance of Learning (AoL) program, the sheer complexity of the task and the need to build and sustain faculty engagement in the process standout as areas of meaningful concern for assessment coordinators (Kuh and Ikenbury, 2009; Kuh and Ewell, 2010).

By way of example, the Association to Advance Collegiate Schools of Business International (AACSB) recently reaffirmed its commitment to Assurance of Learning by calling for the tighter integration of AoL within a school's curriculum management process (AACSB: International Eligibility Procedures and Accreditation Standards for Business Accreditation, 2016, p. 29 \& Standard 8). Consistent with trends in higher education, the adaptation of these standards reflects AACSB's emphasis on innovation and continuous improvement. To fully understand the task of improvement we must also recognize that the AoL process is embedded within a larger and more complex "production" or co-creative environment where stakeholders are increasingly demanding a reasonable return from their investment (The Economist, 2012).

The importance of and challenges related to engaging faculty in the AoL process are well documented in the literature (Emil and Cress, 2014; Garrison and Rexeisen, 2014; Gilbreath, Pueblo and Billington, 2016; Honorée, Davis and Lopez, 2016; Kelly, Tong and Choi, 2010; Martel 2007). Frownfelter-Lohrke, Jones and Marshall (2015) advocate that schools use faculty generated initiatives as a means of organically growing engagement and achieving an institution's AACSB goals. Within this context, organic growth refers to creating a system of rewards and supporting resources that encourages faculty, on their own initiative, to seek out methods for advancing the college's assurance of learning goals. 
In this spirit, a large, private, AACSB accredited business college located in the Midwest piloted a bottom-up, faculty driven study assessing whether lean six-sigma could be used to further the goals of its assurance of learning program. Framed as a case study, a small group of faculty proposed evaluating whether two well-known lean six-sigma outcomes-based tools, the DMAIC procedure, an acronym for define, measure, analyze, improve and control (De Koning \& Demast, 2006) and the congruent model (Nader \& Tushman, 1980), could be used to systematically improve AOL activities. While all problem-solving methodologies have their limitations (Mast \& Lookerbal, 2012), lean six-sigma (LSS) procedures are well understood and have an established track record for improving quality.

\section{Lean Six-Sigma and Higher Education}

The current literature on the use of lean six-sigma tools in higher education has identified several challenges and potential barriers to adoption (Antony, Krishan, Cullen and Kumar, 2012; Kureja, Ricks, Jr., and Meyer, 2009; Jenicke, Kumar and Holmes, 2008). Common issues include the necessity of sponsorship and support by senior leadership, difficulty measuring the exact nature of the educational product, necessity of dealing with long measurement time periods common in higher education assessment protocols, the service nature of education and the overall misalignment of faculty reward systems. Nevertheless, there have been some encouraging adoptions and case studies reported in the higher education literature (Al- Kuwaiti and Vijay, 2015; Holmes, Jenicke and Hempel, 2015; Kukreja, Ricks, Jr. and Meyer, 2009; Pavlovic et al., 2014; Yu and Ueng, 2012).

A common assumption of the current literature, however, is that LSS must be adopted, and therefore supported, by the institution at large. Higher education, unlike most other organizational forms, is characterized by a collaborative partnership between individual faculty and administration. As such faculty research can be viewed as a basic operating unit for the application and integration of lean six-sigma practices. It is our position, now supported by our experience, that it is not necessary for the entire organization to adopt lean six-sigma practices, rather individual faculty can successfully integrate LSS practices into the design and execution of their research. When the research is focused on pedagogical issues relevant to the learning 
goals of the institution, we have a situation where lean six-sigma can directly benefit assurance of learning practices and thereby the institution at-large. We find overall the use of lean sixsigma in research related to assurance of learning outcomes to be responsive to Koch's (2003) concern that most TQM applications have historically been focused on non-curricular activities and not focused on the relevancy or value of the educational experience (Blanco and Berger, 2014).

For those who are unfamiliar with lean six-sigma procedures, the DMAIC refers to a data-driven, lean six-sigma methodology that is intended to systematically improve process quality (George, 2003). The DMAIC can be used to improve both the processes and outcomes associated with assurance of learning. For the benefit of our readers, we organize and report our findings using the framework of the DMAIC model itself.

\title{
First Step - Define the Problem
}

DMAIC is not an implementation method for best practices; it is a method to discover best practices.

\author{
Carl F. Berardinelli
}

Although previous studies have tended to frame LSS applications in higher education from an institutional perspective (Jenicke et al. 2008; Kukreja et al., 2009 and Yu and Ueng, 2012), our research finds that individual faculty can also benefit from using the DMAIC procedure to help inform and guide their research initiatives. Adopting a project to project isomorphic perspective (Miterev, M., Engwall, M., \& Jerbrant, A. 2017), faculty can replicate outcomes between each other rather than a larger, strategic, top-down leadership narrative that might not fit the temporary nature of each class engagement. The case study begins, therefore, with the authors defining the relevant processes and stakeholders who are impacted by our assurance of learning project. The purpose for mapping the domain of a project is to establish program boundaries wherein the scope of a project charter can be developed and agreed upon by appropriate parties. Faculty as stakeholders will assess the needs and outcomes of each assessment through peer to peer discussions yielding a shared insight, assessment competency and capability (Lindkvist, 
2004; Morris \& Geraldi, 2011). This process is similar to defining the scope of a traditional research project but also includes considering the interests of others who will undoubtedly impact our ability to implement relevant findings. As a consequence, we were called upon to consider the perspectives of those who may eventually need to support identified process improvements.

An early output from the DMAIC process is a project charter, typically a short document, that specifies the rationale for the project and identifies stakeholders, objectives, constraints, activities and concerns that are considered within scope, project risks, benefits, costs and assigns accountability $\left(\mathrm{PMBOK}^{\circledR}\right.$ guide, 2013). The motivation for the current project was driven by the expectation that AACSB accredited programs will continually strive to improve their AoL practices and was informed by the experiences of our institution and published research that reported significant challenges continuing to confront program assessment in institutions of higher education. The primary goal of our research was to evaluate the value of using LSS tools to help organize assessment activities. The project was initiated by an individual faculty member and was framed within the context of normal faculty research expectations. The deliverables for the project were defined as a report to the college on the feasible application of LSS to AoL, a report on the findings of the effectiveness of a targeted AoL curriculum innovation and the submission of a peer-reviewed article on the findings. Stakeholders for the project were defined as Department Chairs, the Dean, and the Curriculum \& Assurance of Learning Committees of the College. The authors of this article constitute the accountable parties for the project.

The writing of the project charter highlighted the importance of aligning the purpose of the research and the mission of the college, focused attention on how outcomes from the project might eventually be implemented and caused greater consideration of the needs of, and potential constraints imposed by, the various stakeholders impacted by the project. Unlike previous pedagogical research conducted by the authors, scalability and process improvement became prominent goals for the project.

\section{Second Step - Measure}


The second step of the DMAIC procedure focuses on the collection of data or the measurement of baseline opportunities for improvement. Road-mapping activities (Phaal \& Muller, 2009) are a key benefit to understanding the current system of outcomes (Nadler \&Tuschman, 1980). Faculty through shared baselining efforts (discussion and meetings) can develop tacit understanding of the AoL current processes. The authors began their data collection with a review of the published literature on assurance of learning in business schools. These studies reveal a slow, gradual development of AoL processes as faculty have gained knowledge of assessment practices, adjusted to the new AoL standards, and adopted goals, objectives and assessment methods (Martell, 2007; Kelly et al., 2010).

Faculty resistance to the adoption of the AoL processes has been reported in the literature due to insufficient time and incentives, concern about improper use of assessment results and interference with traditional faculty prerogatives (Pringle \& Michel, 2007; Broker, 2007). By contrast, schools with vibrant AoL systems report high levels of faculty involvement and faculty energy (Garrison and Rexeisen, 2014). Factors contributing to faculty skepticism include that it takes a business school on average 4.2 years to assess the effectiveness of curricular changes and persistent questions as to whether the AoL process leads to improvement in student learning or helps to prepare students for their future business careers. The authors conducted an internal audit of faculty attitudes within the college and the results of the study paralleled the conclusions of the published literature.

We subsequently used Nadler and Tushman's (1980) congruence model as a tool to help contextualize and guide the design of our research. The basic working principle of the congruence model is that an organization's performance is a function of the compatibility or "congruence" of the people working within a given culture and organizational structure for the purposes of accomplishing a given set of tasks. The model is then framed by various inputs (environment, resources \& history) that lead to a set of desired outputs (e.g., a predefined measure of impact such as improvement on a student learning goal).

We used a brainstorming methodology to help identify a range of potential elements for each cell of the model (Figure 1). While the authors' analysis for the current case study focused on the 
AoL program for the college, it became very apparent that the congruence model could also be used to identify opportunities for improvement across many different levels of analysis (e.g., by department, academic program, graduate vs. undergraduate, college/school vs. university, etc.).

\section{Insert Figure 1 Here}

After the model was created it was reviewed by faculty and administrative leadership and then adjusted as needed until agreement was reached. All parties to the process recognized that any given map represents a work-in-process that will need periodic review and adjustment for changes in either internal or external environmental changes. Overall, step 2 helped the authors to exercise greater discipline in using data to systematically inform the identification of performance gaps in the college's AoL program. We also found that the congruence model was a very effective tool for those who prefer visual cues for processing information and making decisions.

For the purposes of illustrating the remaining steps of the lean six sigma methodology, the authors then conducted a study focusing on how a college can improve one of its stated learning goals. Overall the lean six-sigma process heightened the disciplinary emphasis on the speed of data collection and analysis, infer causal relationships, and eventual need to build faculty confidence in the assessment procedures used and overall treatment effectiveness. We

concluded that use of pilot studies in our Assurance of Learning Program were ideally suited for these requirements. Pilot projects are less disruptive, minimize time and expense, and thereby encourage curricular innovation. Pilot projects with encouraging findings can, as needed, be easily scaled into larger studies. Smaller samples, typical of pilot studies, also enable multiple pilot projects to run simultaneously.

\section{$\underline{\text { Third Step - Analyze }}$}

Unlike traditional pedagogical research where faculty attention tends to focus on hypothesis generation or testing, analysis in the DMAIC procedure is grounded in the purposes set forth in the project charter for the study. Throughout the semester faculty can collaborate on 
understanding the stakeholder needs and assessment requirements that could lead to a clear root cause scenario (Longman and Mullins, 2005) for improving the classroom experience and learning. In our case example, the authors were interested in both process improvements to the AoL program and the generation and testing of a promising learning intervention. Consistent with previous findings, the authors observed that the length of time it takes to assess whether a given curriculum change, known as a closing-of-the-loop activity, results in a positive learning outcome is critical to faculty engagement.

Faculty are, of course, notoriously critical of each other's research and there is great value in the scientific enterprise in maintaining the vigor of these critical assessments. Unfortunately, faculty skepticism can serve as a convenient excuse for not implementing an improvement, either in hopes of discovering a better solution or of escaping the inevitable costs associated with change. Higher education can no longer afford to let perfection be the enemy of incremental improvement. Pilot studies by their very nature have the virtue of a more modest pretense, and if results are encouraging, future study provides the opportunity to establish additional empirical support while simultaneously building cultural acceptance. Based on very encouraging results the authors were, by way of example, encouraged to launch a larger and arguably more refined follow-up study on the effectiveness of the learning intervention developed during this project.

\section{Fourth Step - Improve}

By systematically working through the DMAIC the authors developed a better understanding of the interdependencies between identifying opportunities for process improvement (characteristics of our AoL program) and those of process application (measuring and improving performance on specific learning goals). The improvement phase of the DMAIC helped the authors to recognize that Assurance of Learning studies framed using LSS can simultaneously generate useful insights for multiple dimensions of the overall student outcome assessment process. For example, several process improvements were generated within the current study in addition to the specific assessment of the effectiveness of the trial learning intervention. With regard to process improvements, the authors recognized that there is value in retaining data that assesses the process by which outcome assessments are conducted. In the current study, the method used was 
a case analysis of an experiment framed as a pilot study. How piloting within the college can be improved or how best to compare this methodology against other methodologies remains, however, an open empirical question. In the long-run we believe that the systematic piloting of innovation will set the stage in higher education for the strategic development of incubator programs that will help faculty to make more data driven curricular choices and thereby improve faculty energy for and engagement in the AoL process. As noted by one accrediting body:

"Accreditation standards and associated processes should foster quality and consistency, but not at the expense of the creativity and experimentation necessary for innovation. Also, accreditation standards and processes should not impede experimentation or entrepreneurial pursuits; the standards must recognize that innovation involves both the potential for success and the risk of failure. Therefore, when assessing any success or failure, it is key to recognize the importance of experimentation and place a priority on strategic innovation." (AACSB standards, 2016, p. 4).

In this vein, the results of the pilot study suggested where improvements or refinements can be made in both our learning interventions and the methodology that was used to assess the students' learning outcomes. The dual focus on both learning outcome and process revealed that there are many layers in an assurance of learning program and like a Matryoshka doll, process is best understood as a nested phenomenon. The authors found that lean six-sigma provides a set of useful tools whereby organizations and individual faculty can make more meaningful discernments for the purposes of improving program effectiveness.

\section{Fifth Step - Control}

The final step in the DMAIC procedure is control. As with other aspects of the DMAIC, the control phase of the project can operate on multiple levels ranging from ensuring that the gains obtained during the improve step for a given study are retained after the project has ended, to creating a master project management blueprint to ensure the efficient and effective operation of the AoL program. Although the extant literature notes many weaknesses associated with implementing closing-the-loop activities, the authors were impressed how the control phase of 
the DMAIC procedure drew valuable attention to the sustainability of actions taken to close-theloop. Despite substantial progress, however, closing-the-loop continues to be a major challenge for business schools (Martell, 2007; Pringle \& Michel, 2007). While many factors contribute to the difficulties schools are experiencing in closing-the-loop, the complexity of the task and challenges associated with keeping faculty engaged in the AoL process standout as areas of prominent concern (Rexeisen \& Garrison, 2013; Garrison \& Rexeisen, 2014). On further reflection, the authors concluded the issue of sustainability has not been systematically addressed in the literature, at least not with regard to assurance of learning activities.

For example, although the registrar provides a traditional control mechanism for documenting whether a given course is taken and successfully completed, the collective experience of the authors suggests that process of standardizing and then sustaining within-course learning interventions is highly problematic both within and across the curriculum. Faculty objections framed in terms of academic freedom or professional prerogative, the use of adjuncts or faculty teaching a course as a new prep, real time in-class experimentation by instructors, simply forgetting or on-the-fly course adjustments all standout as examples for why "the best of intentions" do not always result in the standard implementation of planned quality improvements. In other words, the ability of the registrar to serve as a control mechanism in education assumes that a common course designation provides a more-or-less standardized learning opportunity.

The authors believe that the writing of quality control documents will help to generate a more pragmatic consideration of alternate control mechanisms, wherein accountability is assigned and process documents (data) are created that will provide a basis for studying and implementing future improvement. In other words, the DMAIC procedure is designed to be a self-correcting methodology. In the current example, problems with control management can themselves be the subject of a quality improvement project.

\section{Summary and Conclusions}


Throughout the process of evaluating the value of using LSS culture and applications to improve our AoL process the authors repeatedly, and often unexpectedly, discovered new insights about the College's AoL program. The discovery of these insights was, in and of itself, intellectually rewarding and therefore stimulated higher levels of engagement in the project. By way of example, the authors observed that although cost effectiveness and impact are stated goals for our AoL program, our College has heretofore assumed that all learning objectives are effectively equivalent in terms of potential impact and associated cost. In other words, the college's learning goals have never been systematically prioritized, neither for the impact on different stakeholders (e.g., potential employers or entering students) nor for the potential return on investment that may result from the implementation of a given curricular adjustment. The authors also realized by extension that neither scalability nor future flexibility have been systematically considered in our closing-of-the-loop activities. Insights, that on reflection, have broader implications for the review and interpretation of traditional pedagogical research.

The authors were encouraged by senior leadership to adopt a "try, test, revise, implement or abandon approach" throughout the execution of our project. Some readers will recognize this as a form of the "fail fast" agile strategy. In the long run we believe that an expanded set of process metrics will need to be developed to more fully assess how success or failure is to be measured as well as metrics on lessons learned. The development and testing of curricular innovations using lean six-sigma tools, and specifically of our assessment of using an incubator/pilot protocol, caused the authors to think more systematically about a wide range of logical connections implied by assurance of learning. For example, the authors became aware that the college has not systematically collected data on how learning goals align with employer expectations (current or future). Future studies are now planned to explore this cross-boundary connection and pursue strategies for improving collaboration.

A common criticism or difficulty of AoL programs is the choice of how to measure learning goals. The experience of our college has been that faculty and/or program directors frequently want to change the measures used to assess students. Although the motivation appears to be a genuine expression of faculty's desire to improve operational measures, a change in instrumentation effectively resets the AoL clock and delays opportunities for developing closing- 
the-loop activities. Generating evidence of continuous improvement remains an important requirement for ongoing accreditation and for maintaining faculty engagement. Our study suggests that a parallel pilot or exploratory project would help minimize this effect and framed within the discipline of a lean six-sigma framework the project could result in more sustainable outcomes.

The author's experience in the current case study suggest that DMAIC framework and congruence model are also useful tools to use when engaging in thought experiments. For example, identifying and prioritizing AoL elements using the congruence model, the practice of writing or mere contemplation of preparing a project charter or control documents can produce constructive insights and opportunities that will serve as a baseline for improving an AoL program.

Based on lessons learned from the current case study, we offer the following recommendations for colleagues seeking strategies to improve their assurance of learning programs:

1. Encourage and support organic faculty initiatives that focus on AoL activities. This should include both process initiatives and the testing of methods specifically designed to improve learning outcomes. Our college uses summer research grants to support and stimulate this type of activity.

2. We recommend encouraging and incentivizing individual faculty to begin experimenting with lean six-sigma. While we admire the aspiration of institutional adoption, we found that individual faculty adoption circumvented or minimized many of the problems that are reported in the literature as being associated with implementing lean six-sigma in higher education. Faculty already familiar with LSS are a logical group to approach as are other faculty who have an interest in pedagogical research related to assurance of learning outcomes.

3. Given the comparative speed and lower cost of pilot studies, we find that their use helps to stimulate innovation and active experimentation. Since individual faculty conducting the research are already engaged and invested in the results, our finding is that it makes further departmental experimentation and adoption easier than if mandated from the top-down.

4. Provide internal forums where faculty can meet and share lessons learned from the AoL focused research. Our college provides a number of opportunities including research and teaching brown bag seminars in addition to a more formal annual research forum held each spring. 
In closing, we find that the use of lean six-sigma tools, such as the DMAIC and congruence models, when adopted and implemented by individual faculty, holds great promise for improving the performance of assurance of learning programs. As such, and by way of example, our case study demonstrates how the use of multiple approaches to continuous improvement, such as lean six-sigma used in combination with AACSB AoL guidelines, benefit both institutional and individual ambition. 


\section{References}

AACSB (2013; last updated January 31, 2016) Eligibility Procedures and Accreditation Standards for Business Accreditation Accessed March 2016 at http://www.aacsb.edu/accreditation/standards/

Al-Kuwaiti, A. and S.A. Vijay (2015). “Appraisal of Students Experience Survey (SES) as a measure to manage the Quality of Higher Education in the Kingdom of Saudi Arabia: An Institutional Study Using Six Sigma Model.” Educational Studies, Vol. 41, No 4, pp. 430-443.

American Management Association (2010). Executives say the $21^{\text {st }}$ century requires more skilled workers. Retrieved from:

http://www.p21.org/storage/documents/Critical\%20Skills\%20Survey\%20Executive\%20Summar y.pdf

Antony, J. (2014). "Readiness Factors for the Lean Six Sigma Journey in the Higher Education Sector" International Journal of Productivity and Performance Management. Vol 63, No. 2, pp. 257-264.

Antony, J., Krishan, N., Cullen, D. and M Kumar (2012). "Lean Six Sigma for Higher Education Institutions (HEIs): Challenges, barriers, success factor, tools/techniques” International Journal of Productivity and Performance Management. Vol 61, No 8, pp. 940 948.

Berardinell, C.F. (2012). “To DMAIC or Not to DMAIC?” Accessed November 2017: http://asq.org/quality-progress/2012/11/back-to-basics/to-dmaic-or-not-to-dmaic.html

Blanco-Ramirez, G. and J.B. Berger (2014). "Rankings, Accreditation, and the International Quest for Quality: Organizing and Approach to Value in Higher Education" Quality Assurance in Education, Vol. 22, No 1, pp. 88 - 104. 
De Koning, H., De Mast, J., (2006) “A Rational Reconstruction of Six Sigma’s Break- through Cookbook" International Journal of Quality and Reliability Management 23 (7), 766-787.

De Mast, Jeroende \& Joran Lokkerbol (2012), “An analysis of the Six Sigma DMAIC method from the perspective of problem solving" Production Economics 139 604-614, Elsevier.

Eaton, J. (2010). Accreditation and the federal future of higher education. Academe, Vol. 94 (5). http://www.aaup.org/article/accreditation-and-federal-future-highereducation?wbcpurpose=Basic\%23sidebar\%23main\#.UZjjbyt35k8 Emil, Serap, and Christine Cress (2014). "Faculty perspectives on programme curricular assessment: individual and institutional characteristics that influence participation engagement." Assessment \& Evaluation in Higher Education 39.5 (2014): 531-552.

Frownfelter-Lohrke, Cynthia, Steven T. Jones, and Jennings B. Marshall. "Adapting Faculty Initiatives to Minimize the Assessment Burden: A Bottom-Up Approach." Journal of the Academy of Business Education 16 (2015): 97.

Garrison, Michael and Rexeisen, Richard J. (2014) "Faculty Ownership of the Assurance of Learning Process: Determinants of Faculty Engagement and Continuing Challenges" Journal of Education for Business, Volume 89, Issue 2, 2014, pages 84-89. DOI:

$10.1080 / 08832323.2012 .761171$

George, ML (2003). Lean Six Sigma for Service: How to Use Lean Speed \& Six Sigma Quality to Improve Services and Transactions. New York: McGraw-Hill.

Gilbreath, Brad, C. O. Pueblo, and Peter Billington. "Helpful Tools for Managing the Assurance of Learning Process." 3 New: Keyword Index-search quicker for papers of interest with keywords (2016): 111.

Hattie, J., Biggs, J., and Purdie, N. (1996). "Effects of Learning Skills Interventions on Student Learning: A Meta-Analysis”, Review of Educational Research, 66(2), 99-136. doi: $10.3102 / 00346543066002099$ 
Higher Learning Commission (2017) "The Criteria for Accreditation and Core Components," https://www.hlcommission.org/Criteria-Eligibility-and-Candidacy/criteria-and-corecomponents.html, criterion 5.C.2.

Holmes, M., Jenicke, L., and J.L. Hempel (2015). "A Framework for Six Sigma Project Selection in Higher Educational Institutions, Using a Weighted Scorecard Approach" Quality Assessment in Education. Vol. 23, No. 1, pp. 30 - 46.

Honorée, André L., Harold E. Davis, and Terri Tará Lopez. "Motivating Faculty Engagement in Assurance of Learning Using the Job Characteristics Mode." Business Education \& Accreditation, Vol. 8(2), p. 1-12, (2016).

Jenicke, L.O., Kumar, A., and M.C. Holmes (2008). "A Framework for Applying Six Sigma Improvement Methodology in an Academic Environment" The TQM Environment. Vol. 20, No. 5, pp. 453-462.

Kelly, C., P. Tong and B-J Choi (2010). "A Review of Assessment of Student Learning Programs at AACSB Schools: A Dean's Perspective" Journal of Education for Business, 85, 299-306.

Kerr, C., \& Phaal, R. (2015, July-August). Visualizing roadmaps: A design-driven approach. Research-Technology Management, 58(4), 45-54.

Koch, J (2006) "TQM: Why is its impact in Higher Education So Small" The TQM Magazine, Vol. 15, No 5, pp. 325-333.

Kuh, G. and S. Ikenberry (2009) "More Than You Think, Less Than We Need: Learning Outcomes Assessment in American Higher Education", National Institute for Learning 
Outcomes Assessment, (October)

http://learningoutcomeassessment.org/documents/fullreportrevised-L.pdf

Kuh, G. and P.T. Ewell (2010) "The state of learning outcomes assessment in the United States", Higher Education Management and Policy, Volume 22/1, pp. 9 - 28.

Kukreja, A., Ricks Jr., J.M., Meyer, J.A. (2009). "Using Six Sigma for Performance Improvement in Business Curriculum: A Case Study" Performance Improvement, Vol. 48, No. 2 , pp. $9-25$.

Lindkvist, L. (2004). Governing project-based firms: Promoting market-like processes within hierarchies. Journal of Management and Governance, 8(1), 3-25.

Longman A., Mullins J. (2005). "The Rational Project Manager: A Thinking Team's Guide to Getting Work Done" Kepner-Tregoe, Wiley Press.

Martell, K. (2007). “Assessing student learning: Are business schools making the grade” Journal of Education for Business, 82, 189-195.

Miterev, M., Engwall, M., \& Jerbrant, A. (2017). "Mechanisms of Isomorphism in Project-Based Organizations.” Project Management Journal, 48(5), 9-24.

Morris, P. W. G. and Geraldi, J. (2011). Managing the institutional context for projects. Project Management Journal, 42: 20-32.

Nadler, D.A. \& Tushman, M.L. (1980) A Model for Diagnosing Organizational Behavior Organizational Dynamics, 9 (2), 35-51.

Orellana, A., Hudgins TL and MR Simonson (2009). "The perfect online course: best practices for designing and teaching", Zheng, and Smaldino (eds) Information Age Publishing

Pavlovic, D., Todorovic, M., Mladenovic, S. and P Milosavljevic (2014). "The Role of Quality Methods in Improving Education Process: Case Study” Serbian Journal of Management, Vol 9, No. 2, pp. 214-230. 
PMBOK® Guide, (2013). A Guide to the Project Management Body of Knowledge, Fifth ed., Project Management Institute.

Pringle, C., \& Michel, M. (2007). "Assessment practices in AACSB accredited business schools" Journal of Education for Business, 82, 202-211.

Rexeisen, Richard J. and Michael Garrison (2013). "Closing-the-Loop in Assurance of Learning Programs: Current Practices and Future Challenges" Journal of Education for Business, 88, pp. 280 - 285. http://www.tandfonline.com/doi/full/10.1080/08832323.2012.697929.

Report to the Secretary of Education, Higher Education Act Reauthorization Accreditation Policy Recommendations (April 2012) Last accessed May 2015, http://www2.ed.gov/about/bdscomm/list/naciqi-dir/2012-spring/teleconference-2012/naciqifinal-report.pdf

The Economist (December 2012), "Not what it used to be; Higher Education," Accessed April 2015: http://www.economist.com/news/united-states/21567373-american-universities-representdeclining-value-money-their-students-not-what-it

Yu, K-T. and R-G Ueng (2012). "Enhancing Teaching Effectiveness by Using the Six-Sigma DMAIC Model”'Assessment and Evaluation in Higher Education, Vol. 37, No.8, pp. 949-961. 
Figure 1

\section{Step 2 - Measure: Organized using the CONGRUENCE MODEL}
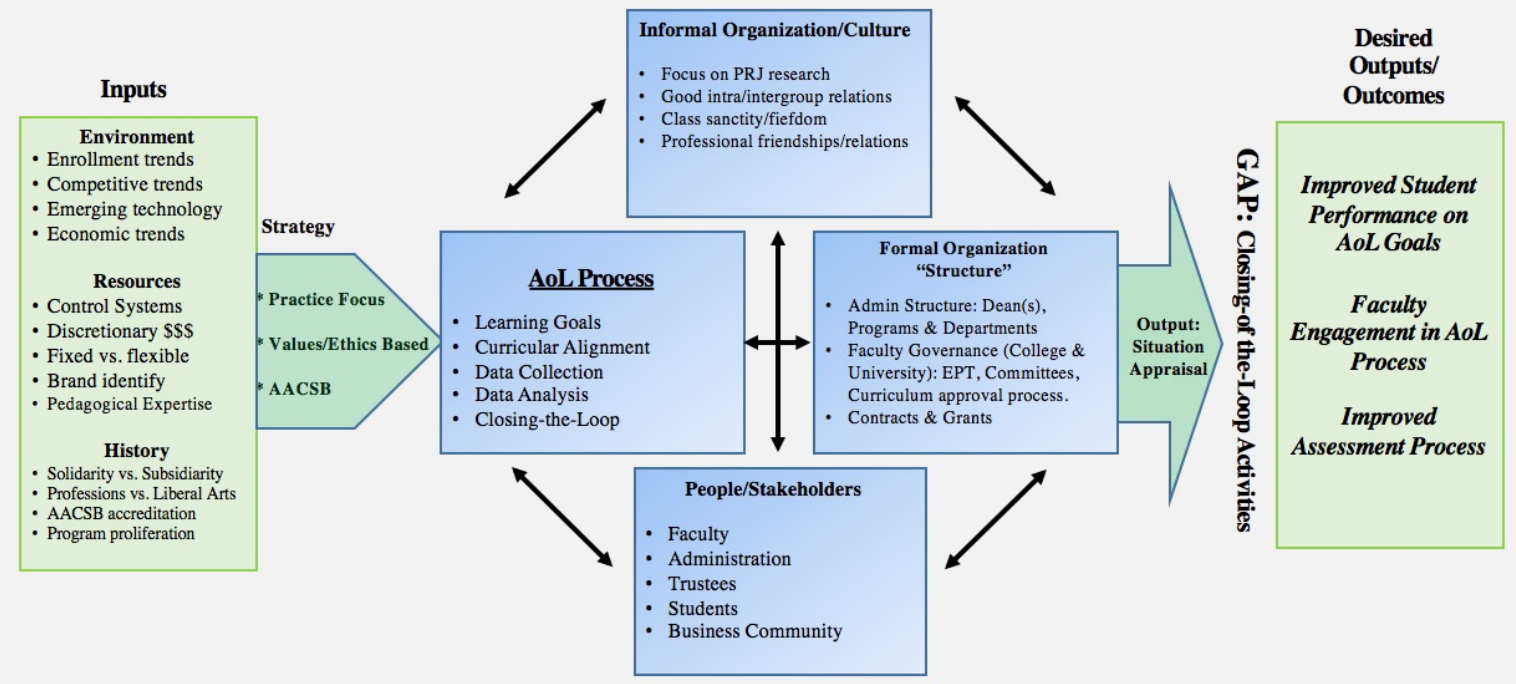